\title{
OBSERVAÇÕES RELACIONADAS À UTILIZAÇÃO DE MONTAGENS ENTRE A FACE POSTERIOR DO PESCOÇO E O COURO CABELUDO NO POTENCIAL EVOCADO POR ESTIMULAÇÃO DO NERVO MEDIANO
}

\author{
G.M. MANZANO*, G. GRONICH**, J.A.M. NÓBREGA***, Y. JULIANO ****, N.F. NOVO ****
}

\begin{abstract}
RESUMO - Foram realizados potenciais evocados por estimulação do nervo mediano no punho, em grupo de voluntários normais. Foram avaliados os componentes que ocorreram entre 12 e $22 \mathrm{~ms}$. Os registros foram feitos com referências cefálicas e não cefálicas. Foi evidenciada maior contribuição do complexo P13-14 na amplitude do

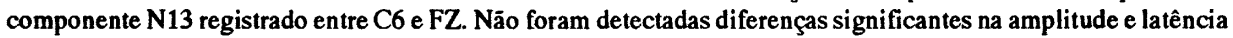
dos componentes N18 e P22 quando avaliados nas montagens FZ-não cefálico e C6-FZ. São discutidas as implicaçōes desses achados para a interpretação clínica dessas respostas.
\end{abstract}

PALAVRAS-CHAVE: potencial evocado, somatossensorial, nervo mediano, componentes subcorticais, referências cefálicas, referências não cefálicas.

Evoked potencial by median nerve stimulation: observations on recordings between posterior neck and scalp references

SUMMARY - Somatosensory evoked potencials after stimulation of the median nerve were obtained in a group of normal individuals. The components obtained between 12 and $22 \mathrm{~ms}$ were analysed with cephalic and non-cephalic references. It is shown that the P13-14 complex is the major contributor for the amplitude of the N13 component recorded with electrodes between the posterior neck and the scalp. It has not been possible to detect differences in amplitude or latencies for the components $\mathrm{N} 18$ and $\mathrm{P} 22$ recorded between FZ-non cephalic and C6-FZ. Implications of these findings to clinical interpretations of the responses are discussed.

KEY WORDS: evoked potencial, somatosensory, median nerve, subcortical components, cephalic reference, non-cephalic reference.

O registro inicial de respostas cervicais, após estimulaçāo do nervo mediano no punho, foi realizado com eletrodos colocados sobre a face posterior do pescoço referenciados a eletrodo colocado sobre o couro cabeludo ${ }^{3,4,8,9,11,13,19,22}$. Registros subsequentes com referências não cefálicas evidenciaram que ambos os eletrodos utilizados inicialmente eram ativos na janela de tempo estudada $^{5,6,10,25}$. Embora fosse inicialmente sugerido que os campos registrados para cada eletrodo representassem faces opostas dos mesmos dipolos ${ }^{10,25}$, estudos subsequentes sugerem que os geradores dos componentes cervicais e dos componentes registrados no couro cabeludo sejam distintos, como proposto inicialmente por outros autores ${ }^{1,6,7,17,20,23}$. Para revisão recente sobre os

Estudo realizado no Serviço de Neurofisiologia Clínica (SNFC) da Disciplina de Neurologia (DN) de Escola de Paulista de Medicina (EPM) e no Serviço de Eletrencefalografia (SEEG) do Instituto de Psiquiatria (IP) do Hospital das Clínicas (HC) da Faculdade de Medicina da Universidade de São Paulo (FMUSP): *Médico, SNFC, DN, EPM; **Médico Supervisor, SEEG, IP, HC, FMUSP; ***Professor Adjunto, DN, EPM; ****Professor Adjunto, Disciplina de Bioestatística, Departamento de Medicina Preventiva, EPM. Durante a realizaçāo desta pesquisa o Dr. G.M. Manzano foi bolsista do CNPq. Aceite: 18-agosto-1993. 
geradores envolvidos ver Manzano \& Nóbrega ${ }^{18}$. Embora, pelo exposto, a utilizaçāo de referência cefálica possa complicar a interpretaçāo clínica dessas respostas, esta abordagem ainda é bastante popular ${ }^{2}$. Por outro lado, estudos recentes sugerem que o emprego de montagens capazes de isolar os componentes provenientes dos diferentes geradores envolvidos sāo também capazes de aumentar a sensibilidade desses testes ${ }^{21,24}$. Um melhor conhecimento da contribuiçăo dos diferentes campos nas montagens com referência cefálica poderia ser útil no sentido de se entender melhor as alteraçōes (ou falta destas) causadas por disfunçōes na via. Se, por um lado, a utilizaçāo de referência cefálica para o estudo dessas respostas apresenta complicaçōes relacionadas com o registro de atividades ocorrendo na mesma janela de tempo, por outro ela permite a avaliação de componentes que não se sobrepōem no tempo. Esta proposta foi feita inicialmente por Lieberson ${ }^{12} \mathrm{e}$ tem sido utilizada em nosso meio ${ }^{14-16}$. Embora esta alternativa seja potencialmente útil, a premissa de que essas atividades sejam registradas sem distorçōes por estas montagens ainda nāo foi totalmente validada.

Baseado no exposto, o presente estudo foi realizado com o objetivo de avaliar a contribuiçăo dos diferentes geradores nas montagens entre a face posterior do pescoço e o couro cabeludo.

\section{MATERIAL E MÉTODOS}

Tabela 1. Amplitude, em microvolts, do componente N13 registrado entre C6-FZ e C6-CAC e a porcentagem representada pela resposta obtida entre C6-CAC em relação àquela obtida entre C6-FZ em 20 individuos normais.

\begin{tabular}{|c|c|c|c|}
\hline & C6-FZ & C6-CAC & $\%$ \\
\hline & 2,6 & 1,3 & 50 \\
\hline & 2,5 & 1,5 & 40 \\
\hline & 1,8 & 1,5 & 17 \\
\hline & 2,6 & 0,8 & 69 \\
\hline & 2 & 1,8 & 10 \\
\hline & 2,6 & 1,2 & 54 \\
\hline & 0,9 & 0,7 & 22 \\
\hline & 1,9 & 0,9 & 53 \\
\hline & 4,3 & 2,1 & 51 \\
\hline & 1,6 & 0,9 & 44 \\
\hline & 2 & 0,6 & 70 \\
\hline & 2,4 & 1,1 & 21 \\
\hline & 3,3 & 0,8 & 76 \\
\hline & 2 & 0,8 & 60 \\
\hline & 2,4 & 1,6 & 33 \\
\hline & 2,9 & 1,5 & 48 \\
\hline & 1,2 & 0,8 & 33 \\
\hline & 2,1 & 1,3 & 38 \\
\hline & 2,4 & 1,6 & 33 \\
\hline & 2,3 & 1,2 & 48 \\
\hline Média & 2,2 & 1,2 & 43,5 \\
\hline
\end{tabular}

Teste de Wilcoxon. (C6-FZ x C6-CAC)

T calculado $=0(p<0,01)$. C6-FZ $>$ C6-CAC.
Foram examinados 24 indivíduos normais, sendo 12 do sexo masculino, com idade variando de 19 a 48 anos (mediana, 33 anos). Todos foram voluntários e concordaram em participar do estudo após explanação detalhada dos procedimentos empregados.

Potenciais evocados, por estimulaçäo do nervo mediano direito no punho, foram obtidos pela aplicação de estímulos elétricos com intensidade igual à soma algébrica em $\mathrm{mA}$ dos limiares motor e sensorial, a uma frequência de 3 estímulos por segundo. Os eletrodos de registro utilizados foram eletrodos comuns de eletrencefalografia, bem como a pasta condutora empregada (bentonite). A impedância entre os eletrodos foi mantida abaixo de $7 \mathrm{~K} 0 \mathrm{hms}$.

Em 20 indivíduos foram obtidos potenciais utilizando-se as seguintes montagens: $\mathrm{C6}$-FZ, C6-face anterior do pescoço (CAC) e FZ-clavícula contralateral (CIC). Os filtros utilizados foram de 25 a $3000 \mathrm{~Hz}$ (3dB). Estas respostas foram obtidas em aparelho AII modelo 900 , com dois canais de registro; o tamanho do bin utilizado foi 100 us.

Em 10 indivíduos foram colhidas respostas com as montagens: $\mathrm{C} 6-\mathrm{CIC}, \mathrm{CAC}-\mathrm{CIC} \mathrm{e} \mathrm{FZ-CIC;} \mathrm{além} \mathrm{disto,}$ foram derivadas a partir destas curvas as respostas provenientes de $\mathrm{C6}-\mathrm{FZeC6}$-CAC, por subtraçäo digital. Os filtros utilizados foram 25 e $1500 \mathrm{~Hz}(-3 \mathrm{~dB})$. Estas respostas foram obtidas em aparelho Nicolet-Pathfinder II, com 256 pontos por curva, num tempo de análise de $50 \mathrm{~ms}$.

Para a análise estatística foram utilizados o teste de Wilcoxon e a análise de variância por postos de Friedman, complementado por teste de comparaçōes múltiplas, quando detectadas diferenças significantes.

\section{RESULTADOS}

Foi detectada a existência de diferença significante nos valores das amplitudes do componente 
Tabela 2. Amplitude, em microvolts, dos componentes N13 e P13 obtidos num grupo de individuos normais com as montagens C6-C1C, CAC-C1C, FZ-C1C, C6-CAC e C6-FZ.

C6-C1C CAC-C1C FZ-C1C C6-CAC C6-FZ

\begin{tabular}{|c|c|c|c|c|c|}
\hline & 1,13 & 0,45 & 2,22 & 1,58 & 3,35 \\
\hline & 0,41 & 0,35 & 1,41 & 0,76 & 1,83 \\
\hline & 0,15 & 0,04 & 1,36 & 0,19 & 1,50 \\
\hline & 0,37 & 0,68 & 0,88 & 1,05 & 1,25 \\
\hline & 0,68 & 0,61 & 2,25 & 1,30 & 2,93 \\
\hline & 0,71 & 0,23 & 1,49 & 0,94 & 2,19 \\
\hline & 0,91 & 1,24 & 1,73 & 2,15 & 2,64 \\
\hline & 0,34 & 0,14 & 1,06 & 0,49 & 1,41 \\
\hline & 1,32 & 0,25 & 1,07 & 1,57 & 2,39 \\
\hline & 0,10 & 0,24 & 1,92 & 0,34 & 2,02 \\
\hline Média & 0,61 & 0,42 & 1,54 & 1,04 & 2,15 \\
\hline
\end{tabular}

Análise de variância por postos de Friedman. (C6-C1C $x$ CAC-C1C $x$ FZ-C1C $x$ C6-CAC $x$ C6-FZ). $X 2$ calculado $=70,24(p<0,0001)$. Comparaçōes Múltiplas. C6-FZ > C6-C1C $(\mathrm{p}<0,05)$. C6-FZ > CAC-C1C (p < 0,05). C6-FZ > C6-CAC $(p<0,05)$. C6-CAC > C6-C1C $(p<0,05)$. C6-CAC > CAC-C1C $(p$ $<0,05)$. FZ-C1C > C6-C1C $(\mathrm{p}<0,05)$. FZ-C1C $>$ CAC-C1C $(p<0,05)$. FZ-C1C $>$ C6-CAC $(p<0,05)$.

N13 obtido nas derivaçōes C6-FZ e C6-CAC, sendo aqueles obtidos utilizando-se FZ como referência significantemente maiores (Tabela 1 e 2). Exemplo de respostas obtidas para esta análise são mostrados na Figura 1.

Foi possivel detectar-se, ainda, diferença significante nos valores absolutos das amplitudes dos componentes $\mathrm{N} 13$ e $\mathrm{P} 13$ obtidos com as montagens $\mathrm{C6}-\mathrm{CIC}$ e CAC-CIC em relaçāo ao componente $\mathrm{P} 13$ oriundo da montagem FZ-CIC (Tabela 2). Exemplo de respostas obtidas para esta análise sáo mostrados na Figura 2.

Com relação aos componentes $\mathrm{N} 18$ e P22, não foi possível detectar diferenças significantes nem com relação a amplitudes nem a latências, utilizando-se as montagens $\mathrm{C6}-\mathrm{FZ} \mathrm{e} \mathrm{FZ-CIC} \mathrm{(Tabela} \mathrm{3).} \mathrm{Exemplo} \mathrm{de} \mathrm{registros} \mathrm{utilizados}$ para esta análise são mostrados na Figura 3.

\section{COMENTÁRIOS}

As análises realizadas no presente estudo sugerem que a contribuição do complexo P13-14, na janela de tempo entre 12 e $14 \mathrm{~ms}$, seja muito mais importante que aquela derivada do componente N13 oriundo da atividade medular segmentar. Estes achados estāo de acordo com as sugestōes iniciais ${ }^{20}$. De fato, estas observaçōes sugerem que a utilizaçäo de montagens desenhadas para o registro de geradores individuais devam aumentar a sensibilidade da técnica, como observado por Restuccia \& Mauguiere 21.

Os dados aqui apresentados sugerem ainda que a análise clínica do componente "N13" na montagem com referência cefálica deva ser feita tendo-se em mente que disfunçōes do(s) gerador(es) do complexo P13-14 devam ser mais importantes na determinaçāo de anormalidades desta resposta. 


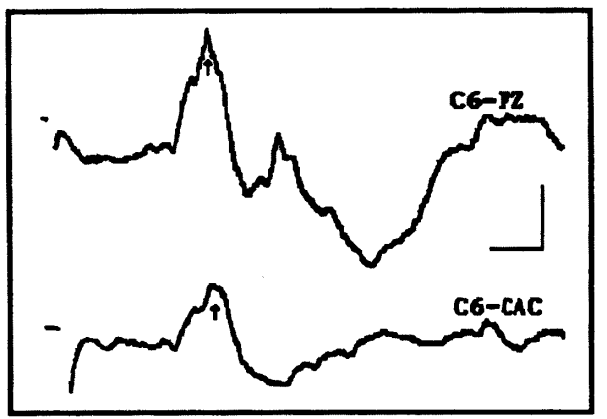

Figura 1. Registro com as montagens C6-FZ e C6-CAC em um voluntário normal. Exemplo de registro utilizado para obtenção dos dados mostrados na Tabela 1. As setas indicam o componente $N 13$ em cada montagem. Calibraçäo 1,25 microvolts e 5,0 milissegundos.

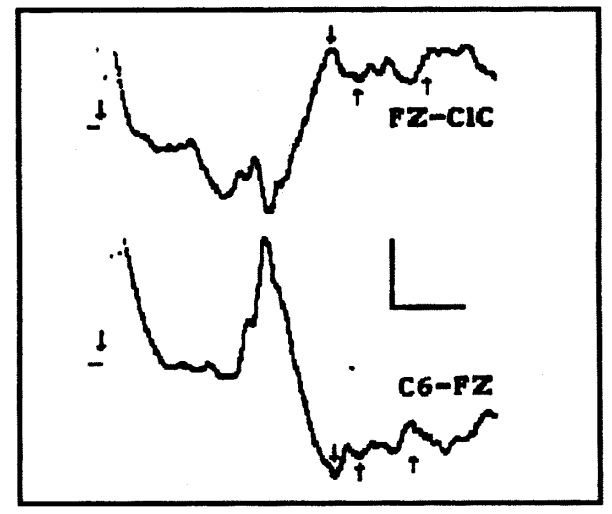

Figura 3. Registro com as montagens FZ-C1C e C6FZ em um voluntário normal. Exemplo de registro utilizado para obtenção dos dados mostrados na Tabela 3. Na curva superior, as setas apontando para baixo mancam o componente $P 22$ e aquelas apontando para cima marcam o componente N18; na curva inferior os mesmos componentes são apontados por setas com direção inversa. Calibração 1,25 microvolts

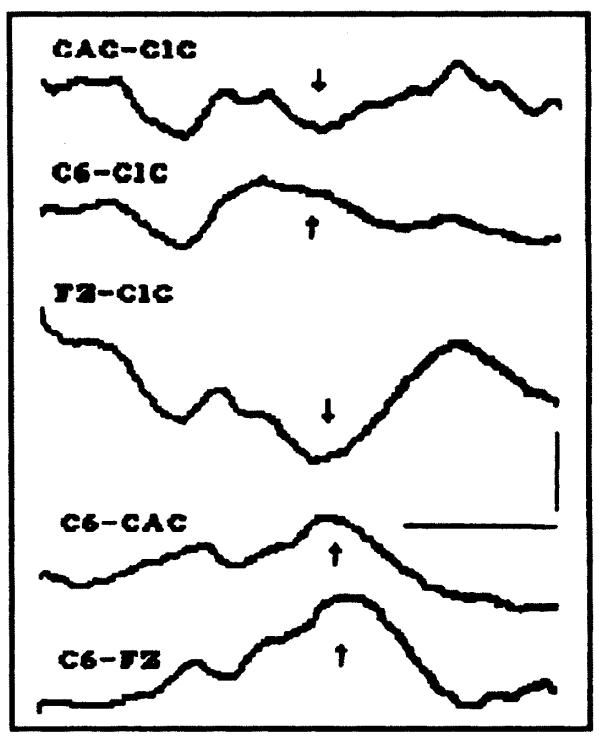

Figura 2. Registro com as montagens C6-C1C, CACC1C, FZ-CIC, C6-CAC e C6-FZ, em un voluntário normal. Exemplo de registro utilizado para obtenção dos dados mostrados na tabela 2. As setas marcam os componentes (Nou P13) na janela dos 13 ms utilizada para análise. Calibraçäo 2,5 microvolts e 4,0 milisegundos

De fato, é a menor contribuição quantitativa dos campos medulares segmentares evidenciada no presente estudo que possibilitaria um aumento de sensibilidade, no estudo de patologias envolvendo a medula cervical com a utilizaçāo da montagem C6-CAC.

Com relaçāo aos componentes acima dos $15 \mathrm{~ms}$ não foi possível detectar diferenças significantes com a utilizaçăo do eletrodo C6 ou uma referência não cefálica. Estas observaçōes reforçam a sugestão de que componentes provenientes dos eletrodos cefálicos possam ser avaliados adequadamente neste tipo de montagem. Nesse sentido, é importante salientar que a possibilidade da utilizaçāo de um só canal de registro, principalmente em nosso meio aonde poucos aparelhos dispöem de mais de 2 a 4 canais de registro, deve ser encarada como opção válida, embora deva-se deixar claro também que a utilização desta abordagem em patologias não foi ainda sistematicamente investigada.

\section{REFERÊNCIAS}

1. Anziska B, Cracco RQ.Short latency SEPs to median nerve stimulation: comparison of recording methods and origin of components. Electroenceph Clin Neurophysiol 1981, 52:531-539.

2. Chiapa KH. Evoked potentials in clinical medicine. Ed 2. New York: Raven Press, 1990.

3. Cracco RQ. The initial positive potencial of the human scalp-recorded somatosensory evoked response. Electroenceph Clin Neurophysiol 1972, 32: 623-629.

4. Cracco RQ. Spinal evoked responses: peripheral nerve stimulation in man. Eletroenceph Clin Neurophysiol 1973, 35:379-386. 
5. Cracco RQ, Cracco JB. Somatosensory evoked potentials in man: far-field potentials. Eletroenceph Clin Neurophysiol 1976, 41:460-466.

6. Desmedt JE, Cheron G. Central somatosensory conduction in man: neural generators and interpeak latencies of the far-field components recorded from neck and right or left scalp and earlobes. Eletroenceph Clin Neurophysiol 1980, 50:382-403.

7. Emerson RG, Seyal M, Pedley TA. Somatosensory evoked potentials following median nerve stimulation: I. The cervical components. Brain 1984, 107:169-182.

8. Goff WR, Allison T, Lyons WB, Fisher TC. Dissociation of auditory and somatic short latency evoked responses recorded from posterior neck region in man. Eletroenceph Clin Neurophysiol 1974, 37:210.

9. Goff GD, Matsumiya Y, Allison T, GoffWR. The scalp topography of human somatosensory and auditory evoked potentials. Electroenceph Clin Neurophysiol 1977, 42:57-76.

10. Jones SJ. Short latency potentials recorded from the neck and scalp following median nerve stimulation in man. Electroenceph Clin Neurophysiol 1977, 43:853-863.

11. Kimura J, Yamada T, Kawamura H. Central latencies of somatosensory cerebral evoked potentials. Arch Neurol 1978, 35:683-688.

12. Lieberson WT. More on one-chanel recording of somatosensory evoked potentials (SEP) of multiple origin. Electroenceph Clin Neurophysiol 1983, 23:607-611.

13. Lieberson WT, Kim KC. The mapping out of evoked potentils elicited by stimulation of the median and peroneal nerves (Abstract). Eletroenceph Clin Neurophysiol 1963, 15:721.

14. Lopes JA, Luccas FJC. Potenciais evocados sómato-sensitivos(PESS) em lesōes afetando plexo, raízes e medula espinal. Reuniōes de Neurofisiologia Clínica 1989, 4:48-77.

15. Luccas FJC, Lopes JA, Plastino FRT, Knobel E. Avaliaçāo neurofisiológica por potenciais evocados sensoriais multimodais em pacientes em coma: aspectos gerais. Arq Neuropsiquiatr 1990, 48:320-328.

16. Luccas FJC, Manzano GM, Ragazzo PC. Potencial evocado sômato-sensitivo cortical: estudo normativo (PESS) (Resumo) In VIII Congresso Brasileiro de Eletrencefalografia e Neurofisiologia Clínica: Temas Livres. São Paulo: Sociedade Brasileira de Eletrencefalografia e Neurofisiologia Clínica 1983, p 36.

17. Lueders H, Lesser R, Hahn J, Klem G. Subcortical somatosensory evoked potentials to median nerve stimulation. Brain 1983, 106:341-372.

18. Manzano GM, Nóbrega JAM. Potencial evocado por estimulação do nervo mediano (PESS), componentes subcorticais. Arq Neuropsiquiatr 1994, 52: 8-19.

19. Mathews WB, Beauchamp M, Small DG. Cervical somatosensory evoked responses in man. Nature 1974, 252:230-232.

20. Mauguiere F. Les potentiels evoqués somesthesiques cervicaux chez le sujet normal: analyse des aspects obtenus selon le siège de l'electrode de reference. Rev EEG Neurophysiol 1983, 13:259-272.

21. Restuccia D, Mauguiere F. The contribution of median nerve SEPs in the funcional assessment of the cervical spinal cord in syringomyelia: a study of 24 patients. Brain 1991, 114:361-379.

22. Shagass C, Schwartz M. Recovery functions of somatosensory peripheral nerve and cerebral evoked responses in man. Electroenceph Clin Neurophysiol 1964, 17:126-135.

23. Stöhr M, Riffel B. Short-latency somatosensory evoked potentials to median nerve stimulation: components N13-P13, N14-P14, P15, P16 and P18 with different recording methods. J Neurol 1982, 228:39-47.

24. Turano G, Jones SG, Miller DH, du Boulay GH, Kakigi R, Mc Donald WI. Correlation of SEP abnormalities with brain and cervical cord MRI in multiple sclerosis. Brain 1991, 114:663-681.

25. Yamada T, Kimura J, Nitz DM. Short latency somatosensory evoked potentials following median nerve stimulation in man. Eletroenceph Clin Neurophysiol 1980, 48:367-376. 